\title{
The Cardiorespiratory Graph in Sleep Apnea and Associated Comorbidities
}

\author{
Carolina Varon ${ }^{1,2}$, Margot Deviaene ${ }^{1,2}$, Dries Hendrikx ${ }^{1,2}$, Sara Van de Putte ${ }^{1}$, Dries Testelmans ${ }^{3}$, \\ Bertien Buyse ${ }^{3}$, Sabine Van Huffel ${ }^{1,2}$ \\ ${ }^{1}$ KU Leuven, Department of Electrical Engineering (ESAT), STADIUS Center for Dynamical \\ Systems, Signal Processing and Data Analytics, Leuven, Belgium \\ 2 imec, Leuven, Belgium \\ ${ }^{3}$ Department of Pneumology, UZ Leuven, Leuven, Belgium
}

\begin{abstract}
The severity of sleep apnea is often assessed using the apnea/hypopnea index (AHI), which is known to be inaccurate in the phenotyping of apnea patients. Hence, better approaches are needed to characterize these patients and to allow cardiovascular risk stratification. In this context, this work studies the cardiorespiratory interactions in patients suffering from both sleep apnea and apnea associated comorbidities by means of graph theory and kernel methods. Results indicate that the total connectivity of the cardiorespiratory graph is significantly $(p<0.01)$ reduced with higher AHI. Moreover, in patients with apnea associated comorbidities, this connectivity appears to be significantly reduced around apnea events. These results are in line with studies that report stronger oxygen desaturations in patients with apnea associated comorbidities, and more unstable control systems, which could be used for a better characterization of apnea patients.
\end{abstract}

\section{Introduction}

Sleep apnea is a sleep-related breathing disorder characterised by repetitive reduced (i.e., hypopnea) or complete (i.e., apnea) cessations of airflow during at least $10 \mathrm{~s}$. The occurrence of these "respiratory events" during sleep has been associated with systemic hypertension and increased sympathetic modulation that in a long-term induce cardiovascular co-morbidities and mortality [1]. Currently, sleep apnea is diagnosed using polysomnography (PSG), which is an overnight sleep test that monitors different physiological signals like heart rate, respiratory effort, and blood oxygen saturation $\left(\mathrm{SpO}_{2}\right)$. From the PSG, different parameters can be derived such as the apnea/hypopnea index (AHI), which is used to assess the severity of sleep apnea and is calculated as the amount of apneas/hypopneas per hour of sleep. For instance, AHI $<5$ is considered normal, $5 \leq \mathrm{AHI}<15$ is mild, $15 \leq \mathrm{AHI}<30$ is moderate, and
$\mathrm{AHI} \geq 30$ is considered as severe. Even though $\mathrm{AHI}$ is one of the most important indices to diagnose sleep apnea, it is well-known that it does not completely correlate with the severity of the disease [2]. Therefore, other information, different than just the amount of events per hour, is needed to better phenotype sleep apnea patients $[2,3]$.

It has been shown that the cardiorespiratory interactions change during sleep apnea events $[4,5]$. Furthermore, in [6] it was shown that patients suffering from apnea-related comorbidities, experienced more severe oxygen desaturations during apnea episodes than patients with similar AHI but without any comorbidity. Hence, the present study investigates if the interactions between heart rate, respiration, and $\mathrm{SpO}_{2}$ are affected in sleep apnea, and if this effect is enhanced by the presence of apnea associated comorbidities. In this way, these interactions could be used to improve the phenotyping of apnea patients and in a longterm improve the prioritization of treatment. These interactions were analysed using an adaptation of the kernel framework proposed in [7] for the construction of physiological graphs. Here, a cardiorespiratory graph is constructed using the heart rate, respiration, and $\mathrm{SpO}_{2}$.

\section{Methodology}

\subsection{Data}

The dataset used in this study consisted of full night PSG recordings of 110 patients referred to the sleep laboratory of the University Hospitals Leuven, UZ Leuven, Belgium. The mean age and BMI of the patients were, respectively, $47.3 \pm 10.6$ years and $29.3 \pm 4.6 \mathrm{~kg} / \mathrm{m}^{2}$, and 100 of them had an $\mathrm{AHI}>15$ while the other 10 had an $\mathrm{AHI}<5$. The latter 10 were referred as the control group and they did not suffer from any apnea-associated comorbidity. The first 100 , on the other hand, were referred as the apnea group and they were divided into two subgroups. One subgroup of 50 patients, referred as the cardiac subgroup, of which 46 suffered from hyperlipidaemia, 40 of hypertension, 5 
from diabetes, 4 had a heart infarct in the past, and 2 a stroke. The other subgroup, referred as the non-cardiac subgroup, consisted of 50 patients who did suffer from apnea but not from any of the aforementioned comorbidities. These patients were matched one-to-one to the patients in the cardiac subgroup according to age, gender, Body Mass Index (BMI) and smoking habits.

From each PSG recording, the single-lead ECG (lead-II) signal was extracted together with the $\mathrm{SpO}_{2}$ and three respiratory signals. The respiratory signals corresponded to the respiratory effort recorded around the thorax $\left(R_{t h}\right)$ and abdomen $\left(R_{a b}\right)$ using inductive plethysmography and the nasal airflow $\left(R_{n}\right)$ recorded using a preassure sensor. All signals were sampled at $500 \mathrm{~Hz}$, and all apnea events were annotated by a sleep specialist according to the AASM 2012 rules [8].

\subsection{Pre-Processing}

The three respiratory signals were first band-pass filtered using a Butterworth filter with cutoff frequencies at $0.05 \mathrm{~Hz}$ and $1 \mathrm{~Hz}$. After that, they were downsampled at 4 Hz. The ECG signals, on the other hand, were used to find the location of the Rpeaks by means of the approach presented in [5]. Then, missed, false, and more importantly, ectopic beats were corrected using the integral pulse frequency modulation (IPFM) model as in [9]. The reason to use this model is that many ectopic beats are expected in the cardiac subgroup, which might interfere with the quantification of the ANS modulation. As a result, the heart rate variability signal (HRV) was extracted. This signal was then resampled at $4 \mathrm{~Hz}$ and band-pass filtered as it was done for the respiratory signals. The last signal was the $\mathrm{SpO}_{2}$, which was only downsampled at $1 \mathrm{~Hz}$.

\subsection{Feature Extraction}

The analysis of the different signals, namely, HRV, $R_{t h}$, $R_{a b}, R_{n}$, and $\mathrm{SpO}_{2}$, was performed using a moving window approach with a window length of $60 \mathrm{~s}$ and an overlap of $50 \mathrm{~s}$. From the power spectral density (PSD) of the HRV and the respiratory signals, the power in the low frequency $(L F: 0.04-0.15 \mathrm{~Hz}$ ) and in the high frequency ( $H F: 0.15-m \mathrm{~Hz}$ ) bands were extracted each $60 \mathrm{~s}$, with $m=\overline{\mathrm{HR}} / 2$ and $\overline{\mathrm{HR}}$ the mean heart rate in the segment. The PSDs were computed using the Welch's algorithm with a Hamming window of $40 \mathrm{~s}$, an overlap of $35 \mathrm{~s}$, and 1024 points.

The $\mathrm{SpO}_{2}$ signal was processed using the same moving window approach but in this case only the mean value was used to characterize each segment.

The last feature was extracted from each ECG segment of $60 \mathrm{~s}$, and it corresponds to the signal quality indicator $(S Q I)$ proposed in [10]. This indicator was used to de-

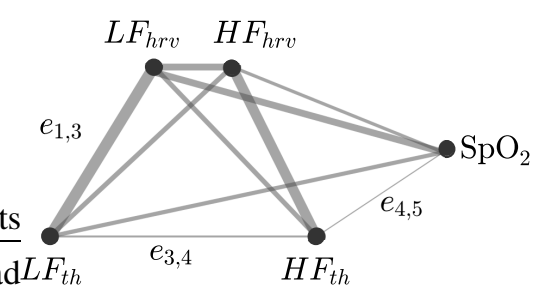

Figure 1. Cardiorespiratory graph $G_{t h}$ constructed using $R_{t h} . \mathcal{V}=\left\{L F_{h r v}, H F_{h r v}, \mathrm{SpO}_{2}, L F_{t h}, H F_{t h}\right\}$. Only 3 edges are indicated for illustration purposes.

tect contaminated segments that could potentially bias the results. It ranges from 0 to 100 and the higher it is, the "cleaner" the ECG segment.

To summarize, this feature extraction approach lead to the derivation of 10 time series, sampled each $10 \mathrm{~s}$ :

- LF and HF powers of HRV: $L F_{h r v}$ and $H F_{h r v}$

- LF and HF powers of $R_{t h}: L F_{t h}$ and $H F_{t h}$

- LF and HF powers of $R_{a b}: L F_{a b}$ and $H F_{a b}$

- LF and HF powers of $R_{n}: L F_{n}$ and $H F_{n}$

- Mean $\mathrm{SpO}_{2}$

- $S Q I$

\subsection{The Cardiorespiratory Graph}

A graph $G=(\mathcal{V}, \mathcal{E})$ consists of vertices $\mathcal{V}=$ $\left\{v_{i}\right\}_{i=1}^{N}$ and edges $e_{i, j} \in \mathcal{E}$, with $e_{i, j}$ the edge between vertices $v_{i}$ and $v_{j}$, and each vertex representing one of the $N$ time series under investigation. In this study, $N=5$ and the vertices were defined as $\mathcal{V}=$ $\left\{L F_{h r v}, H F_{h r v}, \mathrm{SpO}_{2}, L F_{\text {resp }}, H F_{\text {resp }}\right\}$, where $L F_{\text {resp }}$ and $H F_{\text {resp }}$ were derived from one respiratory signal. As a result, 3 graphs were analyzed, each one constructed with a different respiratory signal. Figure 1 illustrates the cardiorespiratory graph $G_{t h}$ constructed using $R_{t h}$. The reason for using only one respiratory signal per graph was to avoid redundant information introduced by the high correlation expected between the respiratory signals.

The cardiorespiratory graph was considered to be an undirected graph, where each edge in the graph has a weight determined by a similarity measure $k_{i j}>0$ and $k_{i j}=k_{j i}$. In fact, this weight indicates the strength of the connection between $v_{i}$ and $v_{j}$. Moreover, how strongly a given vertex $v_{i}$ is connected to the other vertices in the graph is quantified by its degree, defined as $\operatorname{deg}_{i}=\sum_{j=1}^{N} k_{i j}$. As a result, the degree matrix $D$ of the graph can be computed as a diagonal matrix with the degrees $\operatorname{deg}_{1}, \ldots, \operatorname{deg}_{5}$ on the diagonal.

As in [7], the graph was analyzed using a moving window approach, where the topology of the graph remained the same. Here, a window of $60 \mathrm{~s}$ was used to calculate the weights and the degree matrix $D$. Then, a shift of 10 $\mathrm{s}$ was applied each time so that the evolution of the graph 
could be analyzed throughout the night. The weights were computed using the Radial Basis Function defined as

$$
k_{i j}=K\left(x_{i}, x_{j}\right)=\exp \left(-\frac{\left\|x_{i}-x_{j}\right\|_{2}^{2}}{\sigma^{2}}\right),
$$

with $\sigma^{2}$ the kernel parameter, and $x_{i}$ and $x_{j}$ two segments of $60 \mathrm{~s}$ of the time series represented by the connected vertices. As a result, $k_{i j}$ can be seen as the $i j$-th entry of the symmetric kernel matrix (i.e. similarity matrix) $\Omega \in \mathbb{R}^{N \times N}$, with $\Omega_{i j}=k_{i j}=K\left(x_{i}, x_{j}\right)$.

The selection of the kernel parameter $\sigma^{2}$ was done based on the approach presented in [7]. Here, all the kernel matrices computed for each window of $60 \mathrm{~s}$ were concatenated into a large matrix $\tilde{\Omega} \in \mathbb{R}^{N \times m N}$, with $m$ the number of segments (i.e. graphs) analyzed for one recording. This was done for multiple values of $\sigma^{2}$ in the range between 0.01 and 100. For each one of these values, the Shannon entropy $H$ of $\tilde{\Omega}$ was computed and then, the $\sigma^{2}$ for which $H$ was highest was selected as the "optimal" kernel parameter. This was repeated for each recording, so a collection of 110 "optimal" $\sigma^{2}$ values was obtained. After that, the mean value was selected as the final kernel parameter for the analysis of all recordings. The reason for using only one value of $\sigma^{2}$ for all recordings was to guarantee that all graphs were contained in the same space, hence, they could be comparable to each other.

Apart from calculating the weights, the kernel matrix $\Omega$, and the degree matrix $D$ for each window, the overall connectivity of the graph was computed as the average degree $\delta(G)$ defined as $\delta(G)=\frac{1}{N} \sum_{i=1}^{N} \operatorname{deg}_{i}$.

The $\delta(G)$ values and the degrees were then compared among patient groups using the Kruskal-Wallis and multicomparison tests with Bonferroni correction and $\alpha=0.05$. The first comparison was done using the full night recordings, and the mean $\delta(G)$ for the full night was computed using only the clean segments. These clean segments corresponded to those with $S Q I>50$, since they were classified as clean by the algorithm proposed in [10].

The second comparison was done using the apnea events. Figure 2 illustrates the selection of the segments that were used to calculate the mean $\delta(G)$. Note that 7 graphs are considered per apnea since the moving window approach used a window length of $60 \mathrm{~s}$ and a shift of $10 \mathrm{~s}$. For this test, all apneas were taken together and the mean $\delta(G)$ corresponded to the mean connectivity around the events.

\section{Results and Discussion}

The first step was to select the kernel parameter $\sigma^{2}$ to be used for all the experiments. After finding an "optimal" value for each patient, the mean value was selected for all the analysis. This corresponded to $\sigma^{2}=67.4$, and

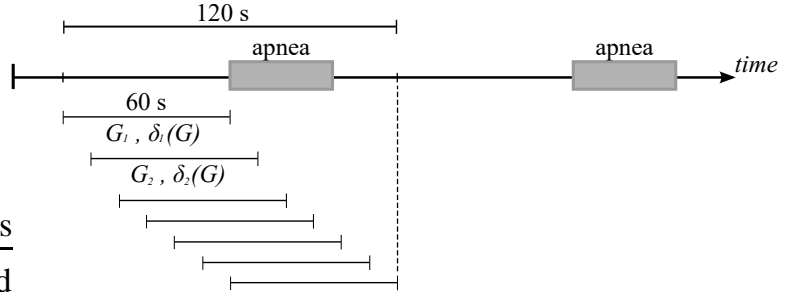

Figure 2. Analysis of the connectivity around apneas. The mean $\delta(G)$ was calculated using only the graphs constructed around the apneas using a window of $120 \mathrm{~s}$.

no differences were found between the values obtained for each patient group. For the control, cardiac, and noncardiac groups, these values were, respectively, $68.2 \pm 4.5$, $66.7 \pm 10.9$, and $67.9 \pm 6.3$.

After selecting the kernel parameter, two experiments were performed. First, the average degree $\delta(G)$ was calculated for the whole night taking into account segments with good quality. Results indicate that the values of $\delta(G)$ decrease with a larger AHI. This can be observed in Figure 3, where it is clear that for increased values of AHI, the connectivity of the graph is significantly lower when compared to the control group $(p<0.01)$. This connectivity, however, is not different for the cardiac group. Therefore, apneas seem to have a stronger long-term effect on the interactions between the signals. The reason to split the AHI at 35 was to obtain a similar amount of patients for each group, namely, 33 and 32 patients with $15 \leq \mathrm{AHI}<35$, and 17 and 18 patients with $\mathrm{AHI} \geq 35$, for the cardiac and the non-cardiac groups, respectively. The results presented here were obtained using $R_{t h}$ but very similar results were obtained with the other 2 respiratory signals.

In $[4,5]$, it was shown that the amount of information transferred from respiratory to heart rate was reduced around episodes of apnea. Hence, it is possible to think that, the connectivity of the cardiorespiratory graph in the full night appears to be lower due to the occurrence of multiple apnea events (i.e. higher AHI). The latter might have a larger effect when averaging the values of $\delta(G)$ for the full night. With this in mind, the second experiment was performed, where only the graphs constructed around the apnea events were considered. At this point, the average degree of the graphs was calculated using the approach depicted in Figure 2. Results indicate that the connectivity of the graph around apneas is significantly lower for the cardiac group, with $p=0.01$. This difference, however, was not present when analyzing different AHIs. Hence, the effect of the cardiac comorbidity appears to be stronger during apneas. No differentiation between the types of apneas (e.g. apneas and hypopneas) was done, hence, future work could focus on whether or not the effect on the connectivity of the graph is different for hypopneas. In addition, the 


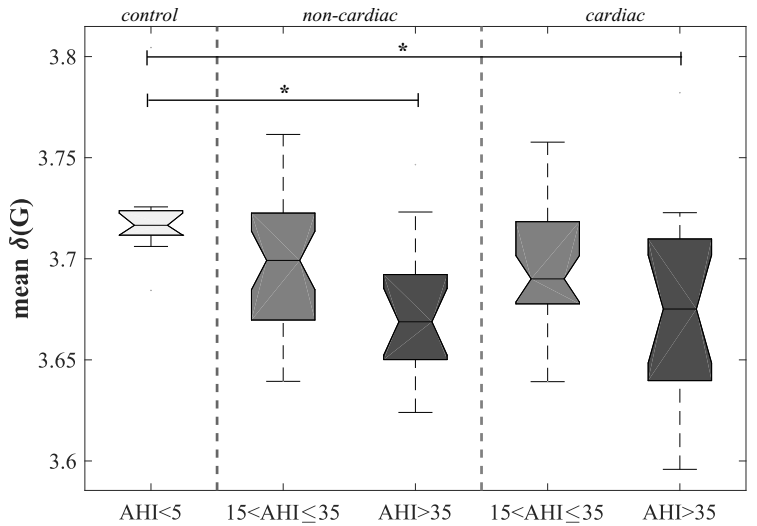

Figure 3. Mean $\delta(G)$ for the full night recordings using only the clean segments. Significant differences are indicated by *.

effect of the duration of the apneic event and the degree of desaturation should also be investigated.

Apart from analysing the total connectivity of the graph, the strength of each independent connection was also studied. This was done for different AHI values and for different patient populations. Results indicate that the connections to the $\mathrm{SpO}_{2}$ vertex were weaker in the cardiac group and this could be associated with the more severe desaturations observed for this patient population [6]. In fact, the lower connectivity in apnea patients might be the result of a weaker control mechanism that allows the autonomic nervous system to react to the occurrence of an apnea episode. As a result, more severe desaturations can occur and a stronger impact in the well-functioning of the heart could take place.

\section{Conclusions}

The results presented in this study suggest that the interactions between the cardiorespiratory signals are affected by the presence of apnea. Furthermore, the response of the cardiorespiratory system to apnea episodes seems to be compromised by apnea associated comorbidities. With this in mind, the quantification of these interactions could be used to better phenotype apnea patients. As a result, an improved diagnosis and treatment could be achieved.

\section{Acknowledgements}

OSA+; imec ICON HBC.2016.0167; European Research Council: The research leading to these results has received funding from the European Research Council un- der the European Union's Seventh Framework Programme (FP7/2007-2013) / ERC Advanced Grant: BIOTENSORS $\left(n^{\circ} 339804\right)$. This paper reflects only the authors' views and the Union is not liable for any use that may be made of the contained information; Carolina Varon is a postdoctoral fellow of the Research Foundation-Flanders (FWO) and Dries Hendrikx is a SB Ph.D. fellow at FWO, Vlaanderen, supported by the Flemish government.

\section{References}

[1] Caples SM, et al. Sleep-disordered breathing and cardiovascular risk. Sleep 2007;30(3):291-303.

[2] Muraja-Murro A, et al. Adjustment of apnea-hypopnea index with severity of obstruction events enhances detection of sleep apnea patients with the highest risk of severe health consequences. Sleep and Breathing 2014;18(3):641-647.

[3] Eckert DJ. Phenotypic approaches to obstructive sleep apnoea-new pathways for targeted therapy. Sleep medicine reviews 2016;

[4] Varon C, et al. Information transfer between respiration and heart rate during sleep apnea. In Computing in Cardiology Conference (CinC), 2016. IEEE, 2016; 845-848.

[5] Varon C, et al. A novel algorithm for the automatic detection of sleep apnea from single-lead ecg. IEEE Transactions on Biomedical Engineering 2015;62(9):2269-2278.

[6] Deviaene M, et al. Assessing cardiovascular comorbidities in sleep apnea patients using spo2. Computing 2017;44:1.

[7] Hendrikx D, et al. Using graph theory to assess the interaction between cerebral function, brain hemodynamics and systemic variables in premature infants. Complexity 2018;.

[8] Berry RB, et al. Rules for scoring respiratory events in sleep: update of the 2007 aasm manual for the scoring of sleep and associated events: deliberations of the sleep apnea definitions task force of the american academy of sleep medicine. Journal of clinical sleep medicine JCSM official publication of the American Academy of Sleep Medicine 2012;8(5):597.

[9] Hernando A, et al. Inclusion of respiratory frequency information in heart rate variability analysis for stress assessment. IEEE journal of biomedical and health informatics 2016;20(4):1016-1025.

[10] Moeyersons J, et al. Artefact detection and quality assessment of ambulatory ecg signals. Submitted to Computer methods and programs in biomedicine 2018;

Address for correspondence:

Carolina Varon

ESAT/STADIUS/KU Leuven

Kasteelpark Arenberg 10, bus 2446, 3001 Leuven, Belgium.

carolina.varon@esat.kuleuven.be 